\title{
Author Index to Volume 89
}

\section{$\mathbf{A}$}

Aara, Naushin 111

Abass, K. Mohammed 498

Abboud, Philippe 549

Abd Elaziz, Khaled M. 260

Abrahão, Jônatas Santos 1142

Abuzaid, Abuzaid A. 1146

Acacio, Sozinho S41

Adamu, Haileeyesus 157

Addiss, David 1186

Adenis, Antoine 564, 1195

Adeyemi, Mitchell O. S13

Adhin, Malti R. 311

Adiossan, Lukas G. 592

Afreen, Sadia 130

Afroz, Dilara 965

Agarwal, Indira 1019

Agudelo, Olga M. 178

Aguilar, Lyla 385

Agunyo, Stella 293

Agwanda, Bernard 1095

Ahmed, Ahmed 1095

Ahmed, Shahnawaz 223, S62

Ahmed, Tahmeed 130, 339

Aidoo, Michael 403

Akanda, Ali 597

Akanda, Ali Shafqat 950

Akhter, Afsana 965

Akinbo, Frederick O. 157

Akinsola, Adebayo S13

Akoko, Daniel 698

Akoko, Daniel O. 875

Akweongo, Patricia 265

Al Baqlani, Said 811

Al Mahrouqi, Salim 811

Al Obaidani, Idris 811

Alam, Munir 597

Alam, Munirul 950

Aldighieri, Sylvain 688

Aldstadt, Jared 1081

Alemayehu, Saba 501

Alexander, James 811

Ali, Doreen 840

Ali, Nabeel A. 965

Alirol, Emilie 145

Allan, Kathryn J. 1095

Allen, Jonathan D. 755

Almazor, Charles P. 617

Almeida, Carlos E. 766

Alonso, Pedro S41

Alonso, Pedro L. S3

Altcheh, Jaime 554

Álvarez, Gonzalo 520

Alves, Ivy J. 1103

Ambrósio, Daniela L. 766

Amornchai, Premjit 794, 797

Amos, Ben 232

Anaseba, Dominic 265

Ando, Katsuhiko 58

Andraghetti, Roberta 688

Andries, Aristomo 1233

Andrus, Jon K. 615, 682

Angelakis, Emmanouil 986

Anglade, Benedict S. 625

Ansah, Evelyn K. 724

Antoniou, Maria 906

Apiwathnasorn, Chamnarn 93

Aquilina, Chloe 535

Aramburu, Carmen 688
Arango, Eliana M. 178

Arango-Bustamante, Karen 937

Archer, W. Roodly 654

Arends, Joop E. 527

Ariza, Juan S. 453

Arman, Alyssa 507

Armien, Blas 489

Arnold, Benjamin F. 130, 238, 251

Arnott, Alicia 188

Ashley, David C. 445

Assis, Felipe Lopes 1142

Assis, Tália Santana Machado 570

Athauda, Indira 742

Atzingen, Marina V. 1103

Avila-García, Miroslava 354

Awine, Timothy 265

Ayele, Berhan 717

Aziz, Nabil 51

Azmi, Ishrat J. 223

Aznar, Christine 1195

\section{B}

Baggett, Henry C. 369

Bair, Ming-Jong 1214

Baker, Kelly K. 214

Baker, Margaret 407

Balajee, S. Arunmozhi 641

Balakrishnan, Kalpana 251

Balasegaram, Manica 1146

Balkew, Meshesha 466

Baltussen, Rob 1146

Bamani, Sanoussi 407

Bandara, Samantha 742

Baqui, Abdullah H. 965

Barnadas, Celine 188

Barrais, Robert 688, 1228

Barreau, Morgane 151

Barrett, Leah J. 531

Barry, Alyssa E. 188

Bartelt, Luther A. 531

Bassat, Quique S41

Bassili, Amal 271

Basurko, Celia 564, 1195

Baubion, Emilie 151

Bazan, Isabel 99

Beaty, Barry J. 385

Beaty, Meaghan K. 385

Becker, Sara J. 380

Becker-Dreps, Sylvia 246

Beckius, Miriam L. 380

Beloto, Albino Jose 1040

Bennett, Adam 840

Berendes, David 647

Berg, Elyssa 540

Berman, Jonathan 557

Bern, Caryn 345

Bernabe-Ortiz, Antonio 229

Bernard, Andrew B. 924

Berrocal, Veronica J. 758

Bertrand, Fabienne 647

Bessong, Pascal O. 531

Bhatti, Zaid S49

Bhengsri, Saithip 369

Bhoomiboonchoo, Piraya 1081

Bhuiyan, Mohammed Ashraful Alam 345

Billeter, Sarah A. 462

Bilodeau, Madeline 781
Birbeck, Gretchen L. 873

Biswas, Kousick S3

Black IV, William C. 385

Blacksell, Stuart D. 308

Blackwelder, William C. 214, S3

Blair, Silvia 520

Blanchet, Denis 564, 1195

Blanco, Victor M. 359

Blevins, Meridith 281

Bliss, Katherine 665

Blum, Annalise G. 411

Boakye, Daniel 540

Bockarie, Moses J. 3

Boelaert, Marleen 1146

Bogoch, Isaac I. 592

Bokhari, Tabassum S49

Bolay, Fatorma K. 11

Boncy, Jacques 641, 654, 1228

Bonini, Marcelo G. 1199

Bonjardim, Cláudio Antônio 1142

Boom, W. Henry 169

Boonsilp, Siriphan 794

Boor, Kathyrn J. 709

Bopda, Jean 916

Borse, Rebekah H. 633

Bossart, Katharine N. 1043

Bougma, Windtaré Roland 407

Bourzac, Kevin M. 308

Boussinesq, Michel 916

Bovier, Patrick 145

Bradley, Mark H. 260

Brault, Aaron C. 205, 474

Breiman, Robert F. 411, 1095, S1, S3, S29

Bretas, Gustavo 311

Brooker, Simon J. 875

Brottet, Elise 211

Brouwer, Kimberly C. 326

Buddhari, Darunee 1081

Bumb, Ram A. 111

Burkhalter, Kristen L. 445

Burns, Stephanie 781

Butler, Thomas 788

Byamukama, Edson 293

C

Cabada, Miguel M. 609

Cabié, André 151

Cabrera, Lilia 229, 507

Cáceres, Mercedes 246

Cadet, Jean 617

Cadet, Jean Ronald 671

Calderon, Maritza 326

Callacondo, David 507

Calvopiña, Manuel 195

Calzada, José 557

Campbell, Karen M. 1066

Camuset, Guillaume 211

Canal, Enrique 1088

Cano, Luz Elena 937

Cantey, Paul T. 16

Cao, Jun 184

Caoili, Janice C. 943

Capitán, Zeuz 557

Cappello, Michael 540

Cardinale, Eric 211

Cardosa, Mary J. 1043

Carme, Bernard 564, 1195 
Carmona-Fonseca, Jaime 178

Carnero, Andrés M. 507

Carothers, Catherine Lem 281

Caruso, Bethany A. 698

Casapia, Martin 99

Cataño, Juan Carlos 1

Cegielski, Peter 943

Cetron, Martin 1226

Chaignat, Claire-Lise 419

Chantra, Somrak 369

Chantratita, Narisara 165, 794, 971

Chapleau, Gina 16

Chappuis, François 145

Charles, Macarthur 625

Charlin, Cyril 211

Chasekwa, Bernard 709

Chávez, Juliana H. 1013

Chelimo, Kiprotich 850

Chen, Huan-Lin 1214

Chen, Hua-Wei 1088

Chen, Lijuan 123

Chen, Ran 23

Chervenak, Keith 169

Chesnais, Cédric B. 916

Chierakul, Wirongrong 369, 797

Ching, Wei-Mei 1088

Chinikar, Sadegh 1135

Chisti, Mohammod J. 223

Chisti, Mohammod Jobayer 339

Chomel, Bruno B. 1219

Choy, Milly M. 1001

Christodoulou, Vasiliki 906

Chu, Brian 407

Chung, Jong-Hoon 119, 1206

Chuquiyauri, Raul 326

Chusri, Sarunyou 899

Clasen, Thomas 426, 875, 1190

Clavijo, Alfonso 1040

Cleaveland, Sarah 1095

Coggeshall, Patricia 501

Cohen, Robert 501

Coleman, Russell E. 1023

Colford Jr., John M. 130, 238, 251

Collazo, Gisela 229, 507

Colton, Leah 462

Colwell, Rita 597

Colwell, Rita R. 950

Concha, Fátima 401

Conn, Jan E. 482

Consolaro, Marcia E. L. 1199

Córdova, Erika 434

Correa, Maria I. 453

Cosivi, Ottorino 1040

Cossio, Alexandra 359

Cota, Gláucia Fernandes 570

Coulibaly, Jean T. 32

Couppié, Pierre 549, 564, 1195

Crespo, Manuel 105

Cui, Liwang 1122

Cui, Yongliang 1122

Cui, Zhuang 1006

Curran, Adrian 105

Currie, Bart J. 365, 367

Curtis, Kurt C. 11

Curtis, Valerie 709

Cutler, Sally J. 1095

\section{D}

D'Anton, Mary 205

D'Alessandro, Umberto 721

da Fonseca, Benedito A. L. 1013

da Rosa, João A. 766

da Silva, João S. 1013 da Silva, Marco T. A. 766

Dahourou, Georges A. 654

Dalaba, Maxwell 265

Damal, Kavitha 482

Dance, David A. B. 973

Darmstadt, Gary L. 965

Das, Mrinalini 1233

Das, Sumon K. 223, S62

Das, Sumon Kumar 339

Datagni, Michael 16

Davenport, Miles P. 850

Davey, Dylan 540

Davis, Jennifer 411

Davis, Timothy M. E. 866

Day, Joanne 205

Day, Nicholas P. 301, 797

Day, Nicholas P. J. 165, 794, 971, 983

Dayan, Gustavo H. 1058

de Abreu, André L. P. 1199

de Aquino, Maria T. P. 1013

de B. Carvalho, Maria D. 1199

de Bedout, Catalina 937

de C. Bronsvoort, B. Mark 1095

de Freitas Nogueira, Betânia Mara 570

de la Ossa, Alberto 453

de Mendonça, Andréa Laender Pessoa 570

de Morais, Zenaide M. 1103

de Oliveira, Thelma F. M. 1013

de Quadros, Ciro 682

de Sadovsky, Ana Daniela Izoton 1058

de Sousa, Marcos Roberto 570

de Zeeuw, Janine 498

Del Brutto, Oscar H. 374

Del Rio Vilas, Victor Javier 1040

del Rosario Nájera-Vázquez, Maria 385

Delatte, Hélène 211

Derado, Gordana 654

Deran, Tong Chor M. 51

Deschamps, Marie Marcelle 671

Desprès, Philippe 211

Deuble, Martin 535

Diarra, Drissa 214

Dietze, Reynaldo 1058

Dil Farzana, Fahmida S62

Dillingham, Rebecca 531

Dodd, Claire 111

Doh, Sanogo S21

Donovan, Ronan M. 924

Dorkenoo, Ameyo M. 16

Dou, Xiangfeng 123

Doumbia, Mama 214

Dow, Steven 973

Dramuke, Patrick 293

Drancourt, Michel 288

Drexler, Naomi 16

Drogoul, Anne Sophie 564

Druce, Julian D. 516

Drury, Patrick 688

Du, Zhizhong 123

Du, Zun-Wei 23

Dufour, Julie 564

Duggal, Nisha K. 205

Duggirala, Sridevi 498

Dujardin, Jean-Claude 750

Dunavan, Claire Panosian 1029, 1032

Duque, Laura 453

Durairaj, Natesan 251

\section{$\mathbf{E}$}

Eberhard, Mark L. 5

Edupuganti, Srilatha 1226

Eidex, Rachel B. 1226

Eisen, Lars 385
Eisenberg, Joseph N. S. 758

El Arifeen, Shames S62

El Arifeen, Shams 965

Elbir, Haitham 288

Elimelech, Menachem 884

Elizalde, Aleix 105

Ellis, Brett R. 1001

Ellis, Esther M. 1001

ElMubark, Wigdan A. 51

El-Setouhy, Maged 260

Emerson, Paul M. 717

Emukah, Emmanuel 578

Endersby, Nancy M. 78

Endy, Timothy P. 1023, 1081

Enrique Zambrana, Luis 246

Epokor, Michael 724

Ershova, Julia 943

Espinoza, Félix 246

Esquela-Kerscher, Aurora 755

Estrada, Gloria 453

Etienne, Carissa F. 615

Etter, Paige 824

Evans, Brian P. 462

Evans, Carlton A. 507

\section{$\mathbf{F}$}

Faix, Dennis J. 991

Fakoli, Lawrence 11

Falcó, Vicenç 105

Fanoy, Ewout B. 527

Farag, Tamer H. 214, S3, S21, S29, S41

Farfan-Labonne, Blanca 354

Farias, Kleber J. S. 1013

Farmer, Paul E. 617

Farrar, Jeremy J. 198

Faruque, Abu S. G. 223, S3, S62

Faruque, Abu Syed Golam 339

Farzana, Fahmida D. 223

Fasabi, Manuel 326

Fatima, Razia 271

Feghali, Karla 501

Feldmeier, Hermann 932

Ferdous, Farzana 223, S62

Ferreira, Érika C. 1199

Ferreira, Paulo Cesar Peregrino 1142

Figueiredo, Luiz T. M. 1013

Fisa, Roser 105

Fischer, Kerstin 11

Fischer, Peter U. 11

Fitter, David L. 633

Fitzpatrick, Christopher 271

Flegg, Jennifer A. 857

Fleming, Fiona 407

Floyd, Katherine 271

Foley, Desmond H. 1117

Forshey, Brett M. 99

Fouché, M. D. Bernadette 654

França, Rafael F. O. 1013

Francois, Jeannot 617, 671

Freeman, Matthew C. 698, 875

Freeman, Nicole 641, 654

Freilij, Héctor 554

Freitas, Simone P. C. 775

Frieden, Thomas R. 615

Fung, Isaac Chun-Hai 633

Fürst, Thomas 592

\section{G}

Gabriel Piñeros, Juan 520

Gage, Kenneth L. 784

Gajadhar, Alvin A. 892 
Galindo-Sevilla, Norma 354

Gallo, Kerry 1186

Galloway, Renee L. 380

Gankpala, Lincoln 11

Gao, Qi 184

Garbes, Pedro 1058

García Bournissen, Facundo 554

García-García, Concepción 1203

Garcia, Melissa N. 996

Garcia, Patricia J. 988

García-Rejón, Julián E. 385

Gardim, Sueli 766

Gardner, Pierce 281

Gaubert-Maréchal, Emilie 549

Gaudart, Jean 1228

Gawoski, John 781

Gayer, Michelle 688

Gaynor, Bruce D. 717

Gebre, Teshome 717

Gebre-Michael, Teshome 466

Gebreselassie, Sintayehu 717

Gedikoğlu, Gökhan 1212

Gelting, Richard 665

George, Christine Marie 138

Gerner-Smidt, Peter 654

Ghalib, Chowdhury Mohammad 345

Gharbi, Myriam 857

Ghiya, Bhikam C. 111

Ghosh, Probir K. 1179

Gibbons, Robert V. 1081

Gilman, Robert H. 326, 507

Gimenes, Fabrícia 1199

Giuberti, Camila 1058

Gler, Ma Tarcela 943

Godoy, Daniel 365, 367

Godsey, Marvin S. Jr. 445

Goenka, Ajit 1033

Goldberg, Tony L. 924

Gomes, Luciana Inácia 570

Gomez-Carro, Salvador 385

Gómez, Gerardo A. 654

González, Angel 937

Goodhew, Brook 345

Goodyear, Andrew 973

Gorchakov, Rodion 996

Goto, Rie 130

Gotuzzo, Eduardo 434

Gouhier, Elise 549

Govha, Margaret 709

Graves, Patricia M. 578

Graves, Stephen 800

Green, Sharone 1081

Greene, Leslie E. 698

Grogl, Max 557

Gruber, Joshua S. 238

Guan, Zengzhi 123

Gubler, Duane J. 1001

Guerin, Philippe J. 857

Guerrant, Richard L. 531

Guevara, Carolina 1088

Guilatco, Ruffy 943

Guillaume, Florence D. 617, 671

Guo, Yayi 869

Gustavo Alonso, Juan Carlos 688

Gute, David M. 950

\section{H}

Haas, Pieter-Jan 527

Habomugisha, Peace 293

Haghdoost, AliAkbar 1135

Haile, Meron 717

Hailemeskal, Meklit Berhan 1186
Haley, Bradd 597

Hall, Eric 1088

Halliday, Jo E. B. 1095

Halsey, Eric S. 99, 1088

Hamer, Sarah A. 924

Han, Eun-Taek 184

Han, Mi Ah 1206

Handel, Ian 1095

Handzel, Thomas 647, 665

Hanf, Matthieu 564

Hang, Jun 501

Hansen, Kristian Schultz 724

Haque, Rashidul 130, 345

Hardcastle, Kathy 1043

Harmon, Jessica R. 445

Harrison, Genelle F. 1117

Harrison, Lisa M. 540

Hartel, Jessica A. 924

Hartskeerl, Rudy A. 1095

Harvey, Kira 16

Hasan, Nur 597

Hashiguchi, Yoshihisa 195

Hashim, Kamal 51

Hassan, Hassan K. 51

Hast, Marisa 641

Hattendorf, Jan 23

Haveman, Nathan 507

Hayashida, Kyoko 116

Hayden, Mary H. 265

Heimburger, Douglas C. 281

Henao, Cesar 453

Hermann, Laura 1081

Herrera, Milagro 489

Heuschen, Florence 688

Hickey, Andrew C. 1043

Hickey, Patrick W. 317

Hidalgo, Marylin 453

Hien, Tran T. 198

Higazi, Tarig B. 51

Hilaire, Isabelle J. 617

Hildenwall, Helena 232

Hill, Audrey A. 365

Hill, Philip C. S13

Hjelle, Brian 489

Hochedez, Patrick 151

Hodgson, Abraham 265

Hoffmann, Ary A. 78

Holding, Penny 1129

Hooper, Pamela J. 407

Hooper, W. Craig 654

Hopkins, Donald R. 5

Horstick, Olaf 198

Hospenthal, Duane R. 380

Hossain, Mohammad J. S13

Huang, Liu-Yu 1006

Huda, Tarique Mohammad Nurul 130

Hudgens, Michael G. 246

Hulangamuwa, Sanjeeva 742

Humphrey, Jean H. 709

Humphries, Debbie 540

Hung, Le Xuan 721

Hung, Nguyen Manh 721

Hung, Nguyen T. 198

Huq, Anwar 597, 950

Husain, Farah 647

Hutubessy, Raymond 419

Iamsirithaworn, Sopon 1066

Imran, Sayeed S13

Inauen, Jennifer 138

Inoue, Noboru 116

Intapan, Pewpan M. 63, 376
Isaakidis, Petros 1233

Islam, Aminul 800

Islam, Shafiqul 950

Islam, Shamim 345

Isoda, Kenichi 58

Ito, Akira 58

Ivers, Louise C. 617

Iwabuchi, Sentaro 58

$\mathbf{J}$

Jackson, Brendan R. 654

Jaenisch, Thomas 198

Jaichapor, Boonsong 1023

Jakhar, Renu 111

Janwan, Penchom 63, 376

Jaramillo, Ernesto 271

Jarman, Richard G. 1081

Jasseh, Momodou S13

Jerome, J. Gregory 617

Jha, Nilambar 145

Jiang, Jin-Yong 23

Jirarojwatana, Ravisara 93

Jirarojwatana, Somchai 93

Johnson, John L. 943

Johnson, Petrina H. 78

Jones, James W. 1023

Jones, Ryan T. 784

Joseph, Gerard A. 641

Joseph, Patrice A. 625

Juin, Stanley 641

Julian, Timothy R. 869

Juliano, Steven A. 482

Junghanns, Thomas 198

Juste, Marc Antoine Jean 671

Jutla, Antar 1231

Jutla, Antarpreet 597

Jutla, Antarpreet S. 950

Jutrakul, Yaowaruk 983

K

Kaan, Jan A. 527

Kaestli, Mirjam 365, 367

Kaewkungwal, Jaranit 369

Kajino, Kiichi 116

Kalayjian, Benjamin C. 1129

Källeståll, Carina 246

Kamau, Edwin 501

Kang, Gagandeep 1019

Kankaew, Prasan 1023

Kanungo, Suman S56

Karanja, Diana M. S. 42

Karbwang, Juntra 737

Kasahara, Kei 460

Kasemsupat, Kriangsak 983

Kass, Philip H. 1219

Kasten, Rickie W. 1219

Katabarwa, Moses 51

Katabarwa, Moses N. 293

Katurahara, Masaki 58

Katz, Mark A. 641

Kawabata, Hiroki 460

Kayange, Petros 162

Kazembe, Lawrence 840

Kazura, James 495, 850, 1037

Keefe, Thomas J. 385

Keenan, Jeremy D. 717

Kelly, Paul 1190

Kenah, Eben 345

Kengluecha, Ampornpan 1023 
Kenyon, Thomas A. 615

Kersgard, Colleen M. 317

Kesav, Praveen 1038

Khakifirouz, Sahar 1135

Khalil, Eltahir A. G. 1146

Khan, Asia S49

Khan, Soroar Hossain S62

Khandelwal, Kanika 111

Khush, Ranjiv S. 251

Kim, Dong-Min 119, 1206

Kim, Heung-Chul 1117

Kim, Seok-Won 119

Kim, Seok Won 1206

King, Charles H. 495

King, Christopher L. 1129

Kinyoki, Damaris 840

Kitkhuandee, Amnat 63

Klein, Terry A. 1117

Knobel, Darryn L. 1095

Kobayashi, Ken-ichiro 58, 1034

Kochel, Tadeusz J. 99, 1088

Köklü, Seyfettin 1212

Kokubo, Yasumasa 58

Komar, Nicholas 474

Komisar, Jack 501

Koné, Siaka 592

Koporc, Kim 1186

Kopydlowski, Karen 557

Kortbeek, Laetitia M. 527

Kosek, Margaret 326, 869

Kosoy, Michael Y. 462

Koster, Frederick 489

Koster, Jacob A. 1043

Kothera, Linda 1154, 1168

Kotloff, Karen L. 214, S1, S3, S13, S21, S29, S56, S62

Kotloff, Karen S41, S49

Kraaij-Dirkzwager, Marleen M. 527

Kraivichian, Kanyarat 899

Kreishman-Deitrick, Mara 557

Kroeger, Axel 198

Krogfelt, Karen A. 960

Kroon, Erna Geessien 1142

Kutsuna, Satoshi 460

Kwong, Jason C. 516

Kyelem, Dominique 407

\section{$\mathbf{L}$}

Labadie-Bracho, Mergiory 311

LaBeaud, Angelle Desiree 495

Lafosse, Elsie 654

Laksono, Ida S. 198

Lakwo, Tom 293

Lalonde, Laura F. 892

Lama, Julio 374

Laman, Moses 866

Lambert, Amy 445

Lammie, Patrick J. 11

Lantagne, Daniele 426

Lapika, Bruno 419

Larrieu, Sophie 211

Lascher, Jonathan 617

Laserson, Kayla F. S29

Lash, R. Ryan 445

Latham, Jonathan R. 223

Laummaunwai, Porntip 63

Leder, Karin 516

Lee, Ji-Woon 119

Lee, John S. 1023

Lee, Jun-Young 688, 1206

Lee, Won-Ja 1117

Lee, Yong-Bok 1206

Lee, Yuan-Kai 1214
Lenhart, Audrey 93

Lepec, Richard 211

Lertiendumrong, Jongkol 369

Leshem, Eyal 641

Levine, Myron M. 214, S1, S3, S13, S21, S29, S41, S49, S56, S62

Levy, Craig E. 474

Li, Chang-Ping 1006

Li, Cheng-Yi 1006

$\mathrm{Li}$, Shen-Long 1006

Li, Weihong 123

Li, Xinyu 123

Li, Zhongze 625

Lietman, Thomas M. 717

Limmathurotsakul, Direk 165, 794, 797, 971, 983

Lim, Tow K. 804

Lin, Audrie 130

Lin, C. D. 1066

Lin, I-Tsung 1214

Lindtjørn, Bernt 466

Linehan, Mary 407

Liu, Lan 246

Liu, Yaobao 184

Lockhart, Gabriella 665, 800

Lodh, Nilanjan 46

Loew, Sabine S. 482

London, Alicia G. 251

Long, Lewis S. 1117

López-Chejade, Paulo 105

Lopez, Martha 609

Lorenz, Camila 928

Loroño-Pino, María Alba 385

Losoya, Arturo 385

Loutan, Louis 145

Louya, Frédéric 916

Lozano-Fuentes, Saul 385

Lu, Feng 184

Luby, Stephen P. 130, 345, 1179

Lucas, Alexander H. 495

Lucien, Mentor Ali Ber 641

Lulitanond, Viraphong 63

Lum, Lucy 198

Lundgren, Ashley 507

Lwamafa, Dennis 292

Lyu, Yanning 123

\section{M}

Ma, Jun 1006

Mabey, David 988

MacDonald, Luke H. 869

Macedo, Paula A. 1168

Macete, Eusebio S41

Machado, Paula R. L. 1013

Machain-Williams, Carlos 385

Mackenzie, Charles D. 51

Maestre, Amanda 178

Magloire, Roc 654, 688

Maguiña, Ciro 401

Maguire, James H. 345

Mahakunkijcharoen, Yuvadee 794

Majewski, Andrew C. 11, 916

Majo, Florence 709

Maleewong, Wanchai 63, 376

Malek, Mohammad Abdul 339, S62

Malhotra, Indu 1129

Malone, LaShaunda L. 169

Mammen, Mammen P. 1081

Mancilla-Ramírez, Javier 354

Mandomando, Inacio S41

Maneeboonyang, Wanchai 794

Manianga, Cele 419

Mann Flueckiger, Rebecca 407
Manna, Byomkesh S56

Manning, Laurens 866

Marfin, Anthony A. 1226

Mariñas, Jamileth 489

Marks, Sara J. 869

Marston, Barbara J. 615

Martin, Diana 345

Martínez, Alejandra 453

Martinez, Eric 198

Martinez, Javier D. 359

Martinez, Leonardo 195, 229, 507

Massebo, Fekadu 466

Mathanga, Don P. 840

Mathieu, Els 16

Matlashewski, Greg 742

Maves, Ryan 1088

Mayanja-Kizza, Harriet 169

Mayen, Friederike Luise 1040

Mayo, Mark 365, 367

Mazarura, Exevia 709

Mbabazi, Pamela Sabina 1186

Mbewe, Edward K. 873

Mbuya, Mduduzi N. N. 709

McCall, Philip J. 93

McCarthy, William F. 557

McLaughlin, Megan M. 174

McMillan-Cole, Audrey C. 1219

McRobb, Evan 367

Meheus, Filip 1146

Mehta, Rajesh D. 111

Mejia, Rojelio 919

Melanson, Vanessa R. 1117

Meléndez, Marlon 246

Meltzer, Martin I. 633

Mende, Katrin 380

Mendoza, Yaxelis 489

Menon, Vipin Kumar 1019

Mercado, Marcela 453

Merten, Sonja 419

Messaritakis, Ippokratis 906

Metcalf, Charlotte J. E. 857

Michault, Alain 211

Mikasa, Keiichi 460

Mikhailov, Alexei 1186

Milbrath, Meghan O. 758

Miller, R. Scott 1023

Mintz, Eric 214

Mintz, Eric D. 641, 654, S29

Miri, Emmanuel S. 578

Missamou, François 916

Moe, Christine L. 698

Mohamed, Hanan A. 51

Mohareb, Emad 991

Moise, Colette Guiteau 625

Moke, Fenny S29

Molina, Israel 105

Monath, Thomas P. 1225

Mondal, Dinesh 339

Monteville, Marshall R. 991

Montoya, Viviana 453

Moonga, Ladslav 116

Moore, David 988

Moore, Sandra 1228

Moormann, Ann M. 850

Morakote, Nimit 376

Morgah, Kodjo 16

Morgan, Douglas R. 246

Moroni, Samanta 554

Morrill, John 495

Morrison, Amy C. 99

Moscatelli, Guillermo 554

Moschella, Samuel 781

Moses, Prabhakar Devarajan 1019

Mosher, Aryc 578

Mostafavi, Ehsan 1135 
Mtei, Frank 232

Mtove, George 232

Muchiri, Eric 495

Mueller, Ivo 188

Muiruri, Samuel 495

Mulligan, Mark J. 1226

Munasinghe, Asoka 742

Mungai, Peter 1129

Muñoz, Carlos 489

Muok, Erick M. O. 42

Murphy, Jennifer 647

Murphy, Sean C. 824

Murray, Clinton K. 380

Murray, Kristy O. 996

Murrell, Ebony G. 482

Musa, Ahmed M. 1146

Mutasa, Kuda 709

Mutengo, Mable M. 46

Mwansa, James C. L. 46

Mwinzi, Pauline N. M. 42

\section{$\mathbf{N}$}

N'Gbesso, Yves K. 32

N'Goran, Dje N. 592

N'Goran, Eliézer K. 32, 592

N'Guessan, Nicaise A. 32

Na-Bangchang, Kesara 737

Nacher, Mathieu 549, 564, 1195

Nadjm, Behzad 232

Najjuka, Anne 232

Nakamura-Uchiyama, Fukumi 58, 1034

Nakao, Ryo 518

Namangala, Boniface 116, 518

Nasci, Roger 205

Nascimento, Ana L. T. O. 1103

Nash, Theodore E. 919

Nasrin, Dilruba S3, S13, S41, S49, S56, S62

Nataro, James P. 214

Navaratne, Chaturi 742

Nawa, Yukifumi 63

Nelms, Brittany M. 1154, 1168

Nelson, Kara 238

Newman, Mercy J. 960

Newman-Gerhardt, Shoshana 495

Newton, Samuel 540

$\mathrm{Ng}$, Reuben 884

Ng'ang'a, Zipporah W. 42

Ngure, Francis M. 709

Nhabanga, Arnaldo S41

Nhacolo, Ariel S41

Nhalungo, Delino S41

Nhampossa, Tacilta S41

Nicholson, William L. 445

Nienhuis, Willemien A. 498

Nieto, Javier 557

Nieto, Melissa 557

Nisalak, Ananda 1081

Nisar, Muhammad Imran S49

Nishiguchi, Takeshi 58

Nizame, Fosiul A. 1179

Njenga, M. Kariuki 1095

Njoku, Chidiebere 578

Nkwocha, Omeni 578

Noedl, Harald 737

Noor, Abdisalan 840

Noppakun, Nopadon 899

Noriega, Fernando 1058

Norton, Robert 535

Nosten, François 308

Ntais, Pantelis 906

Núñez, Heydy 489

Nuñez-Ayala, Guadalupe 385

Nuruzzaman, Md. 1179
Nwankwo, Lawrence 578

Nwodu, Kenrick 578

Nwoke, Bertram E. B. 578

Nyaaba, Gertrude 265

Nygren, Benjamin 654

Nygren, Benjamin L. 641

\section{$\mathbf{0}$}

O'Guinn, Monica L. 1023

O'Reilly, Ciara E. 214, S29

Obasi, Andrew 578

Obeng-Nkrumah, Noah 960

Oberhelman, Richard 229, 507

Obor, David S29

Ochola, Elizabeth A. 42

Ocquidant, Philippe 211

Odhiambo, Frank S29

Oguttu, David 293

Ohnishi, Kenji 58, 1034

Okaka, Christopher E. 157

Okoth, George 411

Olack, Beatrice 1095

Olive, Claude 151

Oliveira, Carlo J. F. 1013

Oliveira, Danilo Bretas 1142

Oliveira, Edward 570

Omansen, Till F. 498

Omore, Richard S29

Omoregie, Richard 157

Onapa, Ambrose 293, 407

Onwuchekwa, Uma S21

Ooi, Winnie 781

Oosterheert, Jan Jelrik 527

Oparaocha, Elizabeth 116

Ostyn, Bart 750

Otali, Emily 924

Otchere, Joseph 540

Oteo, José A. 1203

Ouattara, Mamadou 592

Oyier, Beryl 411

Ozaki, Masayo 345, 578

\section{$\mathbf{P}$}

Pahissa, Albert 105

Panchalingam, Sandra 214

Pandurang Bawikar, Shyam 811

Pandya, Rajul 265

Panella, Nickolas A. 445, 474

Paniagua, Margarita 246

Pape, Jean W. 625, 671

Paré, Alain Brice 407

Parikh, Sunil 824

Paris, Daniel H. 301

Parsons, Michele B. 641

Pascale, Juan M. 489

Patel, Minal K. 811

Patrick, Molly 647, 665

Patterson, Amy 578

Payne, Amanda B. 654

Paz, Hector 557

Peacock, Sharon J. 165, 794, 797, 971, 983

Peck, Mireille 671

Peeling, Rosanna W. 988

Peletz, Rachel 1190

Pelletreau, Sonia 11

Pelloso, Sandra M. 1199

Pelly, Tom F. 507

Pelzman, Jamie 265

Peña, Rodolfo 246
Peñataro, Pablo 326

Pereira-Lourenço, Ariane S. 775

Pérez, Jorge E. 453

Pérez-Hoyos, Santiago 105

Pérez-Lu, José E. 988

Perlada, David E. 588

Perodin, Christian 671

Peters, Clarence J. 495

Petravic, Janka 850

Phosuk, Issarapong 376

Phua, Jason 804

Phumee, Atchara 899

Piarroux, Martine 1228

Piarroux, Renaud 1228

Pickering, Amy J. 411

Pinedo, Silvia Rengifo 869

Pinkevych, Mykola 850

Pinto, Bruna Fernandes 570

Pion, Sébastien D. S. 916

Poncelet, Jean Luc 688

Porco, Travis C. 717

Porter, Chad K. 991

Portilla, Carmencita 520

Portillo, Aránzazu 1203

Poupalos, George 906

Prabhakar, Sudesh 1038

Prajapati, Vijay Kumar 750

Pratlong, Francine 906

Prendergast, Andrew J. 709

Prescott, William R. 824

Price, Erin P. 365, 367

Primack, Aron 281

Prince, Sabine 671

Pruckler, James M. 654

Purdy, Mark 819

\section{Q}

Qadeer, Ejaz 271

Qiu, Feiyou 169

Qiu, Yongjin 518

Quadri, Farheen S49

Quagraine, Josephine 540

Quintó, Llorenç S41

\section{$\mathbf{R}$}

Rabello, Ana 570

Raharimanga, Vaomalala 932

Rahman, Kazi Mizanur 345

Rahman, M. W. 345

Rahman, Sheikh Masudur 138

Rai, Madhukar 750

Rajkumar, Paramasivan 251

Rakers, Lindsay 578

Ram, Pavani K. 411

Ramamurthy, Thandavarayan S56

Ramaprabha, Prabhakar 251

Ramaswamy, Padmavathi 251

Ramírez-Hernández, Alejandro 453

Ramírez-Ramírez, Alicia 354

Ramos-Peña, Yasnina 229

Ramzy, Reda M. R. 260

Ranasinghe, Shalindra 742

Ransom, Janet 557

Raoult, Didier 288, 986

Raqib, Rubhana 130

Ray, Isha 238

Ray, Sayantan 817

Raymond, Max 617

Rebaudet, Stanislas 1228 
Rebollo, Maria P. 3

Reeder, John C. 188

Reisen, William K. 1154, 1168

Restrepo, Angela 937

Reyburn, Hugh 232

Reyes, Julissa 892

Reygadas, Fermin 238

Rheingans, Richard 875

Rheingans, Richard D. 698

Rholl, Drew A. 973

Ribera, Esteban 105

Richard, Vincent 932

Richards, Allen L. 51, 308

Richards, Frank O. 578

Richardson, Jason H. 1117

Richardson, Leisha J. 365

Riche, Claudia T. 625

Riddle, Mark S. 991

Riera, Cristina 105

Ritchie, Scott A. 78

Robinson, Matthew 819

Rocha, Cláudia S. 766

Rocha, Claudio 99

Rocha-Brischiliari, Sheila C. 1199

Rodrigues, Patricia Marques 1058

Roess, Amira A. 965

Roger, Amaury 210, 564

Rogier, Christophe 932

Rosanas-Urgell, Anna 721

Rothman, Alan L. 1081

Rouzier, Vanessa 671

Roy, Sharon L. 5

Roy, Subhasis S56

Roy, Sumitro 1179

Royal, Joseph 991

Rublin, David 819

Rueda, Leopoldo M. 1117

Ruiz-Matus, Cuauhtémoc 682

Ruiz-Tiben, Ernesto 5

Ryan, Edward T. 610

\section{S}

Saavedra-Rodriguez, Karla 385

Saboori, Shadi 698

Sack, R. Bradley 597, 950

Sacoor, Charfudin S41

Saha, Debasish S3, S13

Sainte Marie, Dominique 564

Saiprom, Natnaree 794

Saito, Mayuko 507

Sako, Yasuhito 58

Salam, Mohammed Abdus 339

Salgueiro, Fabián 554

Saliba, Juliana Wilke 570

Salim Al Busaidy, Suleiman 811

Salotra, Poonam 111, 750

Salvador, Fernando 105

Samie, Amidou 531

Samuel, Roshini 178

Sánchez, Deyanira 489

Sanghvi, Tina 1179

Sangmaneedet, Somboon 462

Sankatsing, Sanjay U. C. 527

Sanogo, Doh 214

Sanpool, Oranuch 376

Sanprasert, Vivornpun 899

Santa-Olalla, Patricia 688

Santibáñez, Sonia 1203

Santos-Mallet, Jacenir R. 775

Sanz, M. Mercedes 1203

Saranchuk, Peter 1233

Saravia, Nancy Gore 359

Sarenje, Kelvin 1190
Sarkar, Rajiv 1019

Sarovich, Derek S. 367

Satoskar, Abhay R. 111

Satti, Hind 174

Savage, Harry M. 445, 1154, 1168

Sawanyawisuth, Kittisak 63, 376

Scalmanini, Jenna 411

Schaetti, Christian 419

Schafrick, Nathaniel H. 758

Schneider, Maria Cristina 1040

Scholte, Ernst-Jan 527

Schulze Schwering, Markus 162

Schwab, Kellogg J. 869

Schweizer, Herbert P. 973

Scott, Charles 557

Scott, Thomas W. 99, 1066, 1081

Seas, Carlos 401

Secor, W. Evan 42

See, Kay C. 804

Seethorn, Nongluk 737

Segura-Cervantes, Enrique 354

Seiferth, Robyn 205

Seneviratne, Kamal 742

Senn, Nicolas 188

Seung, Kwonjune J. 174

Severe, Karine 625, 671

Severe, Patrice 670

Sevilleja, Jesus Emmanuel 531

Sharma, Sanjib K. 145

Sharma, Smriti 750

Sharples, Katrina S13

Shelite, Thomas R. 301

Shewchuk, Tanya 857

Shiff, Clive J. 46

Shott, Joseph P. 824

Siba, Peter 188

Siba, Peter M. 866

Sibley, Carol Hopkins 857

Sifaki-Pistola, Dimitra 906

Silairatana, Santi 983

Silisouk, Joy 973

Silué, Kigbafori D. 592

Silva, Ositha 742

Sima, Laura C. 884

Simiyu, Elses W. 42

Simmons, Cameron P. 198

Simms, Benjamin T. 540

Simon, Anna 1019

Simon, Stephane 564

Simunyama, Martin 1190

Simuyandi, Michelo 1190

Singhasivanon, Pratap 794

Siqueira, Gabriela H. 1103

Siriyasatien, Padet 899

Sivanantharajah, Sundaramoorthy 742

Smit, Pieter W. 988

Smythe, Lee D. 797

Snow, Robert W. 840

Soares, Edson G. 1013

Soares Magalhaes, Ricardo J. 1006

Socolovschi, Christina 986

Sodahlon, Yao 16

Sonthalia, Nikhil 817

Sonthayanon, Piengchan 797

Sosa, Néstor 557

Souza, Raquel P. 1199

Sow, Samba O. 214, S3, S21

Spadafora, Carmenza 557

Spitzer, Martin S. 162

Spratt, Brian G. 365, 367

Srikanth, Padma 251

Srikiatkhachorn, Anon 1081

Stauss-Grabo, Manuela 932

Steenland, Maria W. 641

Stein, Catherine M. 169

Steinmann, Peter 23
Stenos, John 800

Stewart, V. Ann 824

Stienstra, Ymkje 498

Stoddard, Steven T. 99

Stoller, Nicole E. 717

Stoltzfus, Rebecca J. 709

Strange, Linda 973

Stuckey, Matthew J. 1219

Sudharsanam, Suchithra 251

Suesdek, Lincoln 928

Sugimoto, Chihiro 116, 518

Sun, Hai-Long 1006

Sun, Yulan 123

Sundar, Shyam 750

Suntornsut, Pornpan 983

Suksawat, Fanan 462

Sur, Dipika S3, S56

Suzuki, Yasuhiko 116

Switzer, Alexandra D. 1219

\section{$\mathbf{T}$}

Tadesse, Zerihun 717

Takano, Ai 460

Takiya, Daniela M. 766

Talkington, Deborah F. 641, 654

Talukdar, Arunansu 817

Talukder, Kaisar A. 223

Tamboura, Boubou 214

Tami, Adriana 198

Tampanya, Vich 899

Tandhavanant, Sarunporn 165, 971

Tanganuchitcharnchai, Ampai 308

Tanwisaid, Kittisak 369

Tao, Li 169

Tappero, Jordan W. 615, 654

Tayapiwatana, Chatchai 63

Tebar, Silvia 105

Teerawattanasook, Nittaya 165, 971

Teng, Jessica E. 617

Ternier, Ralph 617

Terryah, Shawn 540

Thabit Al Awaidy, Salah 811

Thaipadungpanit, Janjira 794

Thalmann, Claudia M. 1043

Thammapalo, Suwich 1081

Thamthitiwat, Somsak 369

Thanchomnang, Tongit 376

Thang, Ngo Duc 721

Thanh, Pham Vinh 721

Tharnpoophasiam, Prapin 794

Théodose, Rafaelle 151

Thiel, Bonnie 169

Thielecke, Marlene 932

Thiemann, Tara 1168

Thomas, Yolanda 281

Tian, Lili 123

Tikmani, Shiyam Sunder S49

Tilley, Drake H. 1088

Tio, Phaik-Hooi 1043

Tisayaticom, Kanjana 369

Tobón, Angela Maria 937

Tobon-Castaño, Alberto 520

Todd, Jim 232

Tomashek, Kay M. 198

Torres, Lorena L. 988

Torres, Scott 326, 407

Torres, Sonia 326

Traore, Mamadou Oumar 407

Trindade, Giliane de Souza 1142

Trongtokit, Yuwadee 93

Trujillo, José 489

Tuah, Wilson 498

Tuinsma, Marjon 407 
Tukahebwa, Edridah M. 293

Tukesiga, Ephraim 293

Turell, Michael J. 1023

Turner, Claudia 308

Turner, Paul 308

Twum-Danso, Kingsley 960

\section{$\mathbf{U}$}

Ud-Din, Abu I. M. S. 223

Ukaga, Chinyere N. 578

Ullman, Diane 557

Unicomb, Leanne 130, 1179

Unnasch, Thomas R. 51, 293

Unoba, Dickson 293

Utzinger, Jürg 23, 32, 592

Uys, Leana R. 873

\section{V}

Valbuena, Gustavo 453

Valenciano, Marta 688

Van Alphen, Dana 688

van den Eede, Peter 721

Van der Stuyft, Patrick 93

van der Werf, Tjip S. 498

Van Eijk, Anna Maria S3, S21, S29

Van Hong, Nguyen 721

van Oosterhout, Joep J. 162

Van Overmeir, Chantal 721

Vanaerschot, Manu 750

Vanlerberghe, Veerle 93

Vantilcke, Vincent 1195

Vasconcellos, Silvio A. 1103

Vélez, Carolina 453

Venkatesan, Meera 857

Venzal, José M. 1203

Verdier, Rose Irene 671

Vermund, Sten H. 281

Vertefeuille, John 641

Vetter, Sara M. 784

Vibhagool, Asda 899

Vicari, Andrea S. 682

Victor, Nadia 617

Vigilato, Marco Antonio Natal 1040

Vignier, Nicolas 151

Vilcarromero, Stalin 99

Vinetz, Joseph M. 326

Vulule, John 850, S29
VY, Vishnu 1038

Vythilingham, Indra 721

\section{W}

Wackwella, Hasini 742

Walker, Damian G. S3

Walker, David H. 301

Walsh, Frank 293

Wang, Bao-Long 1006

Wang, Baomin 1122

Wang, Min 1122

Wang, Quanyi 123

Warner, Tokesha L. 281

Warner, Circle A. 531

Watthanaworawit, Wanitda 308

Weaver, Scott 197

Weber, David J. 246

Wei, Kuangyi 819

Weigel, Jonathan 617

Weil, Gary J. 11, 260, 916

Weina, Peter J. 501

Weisenberg, Scott A. 588

Weiss, Adam 5

Weiss, Mitchell 419

Were, Vincent S29

Wernsdorfer, Walther H. 737

Whitcombe, Elizabeth 597

White, A. Clinton Jr. 609

Whitty, Christopher J. M. 724

Wickremasinghe, Rajitha 742

Wickremasinghe, Renu 742

Wilde, Henry 899

Wilkerson, Richard C. 1117

Williamson, John S29

Wills, Bridget A. 198

Wilson, Mark L. 758

Wilson, Michael 540

Winch, Peter J. 965, 1179

Winkler, Mirko S. 32

Withers Jr., P. Craig 5

Won, Kimberly Y. 11

Wongkham, Chaisiri 63

Wongsuvan, Gumphol 165, 971, 983

Woodworth, Michael 625

Wrangham, Richard W. 924

Wright, Peter F. 625, 671

$\mathrm{Wu}$, Chia-Hsien 1214

Wu, Fang-Wei 23

Wu, Yukun 214, S3, S62

Wuthiekanun, Vanaporn 165, 794, 797, 971, 983
Xiang, Jeannie 205

Xiao, Lihua 157

$\mathbf{X}$

\author{
Y \\ Yactayo, Sergio 434 \\ Yadon, Zaida Estela 1040 \\ Yamasaki, Hiroshi 63 \\ Yan, Chris 996 \\ Yan, Guiyun 1122 \\ Yanagida, Tetsuya 58 \\ Yanow, Stephanie K. 178 \\ Yao, Azragnou J. 592 \\ Yap, Peiling 23 \\ Yeap, Heng Lin 78 \\ Yeo, Leong L. 804 \\ Yeung, Shunmay 724 \\ Yılmaz, Bülent 1212 \\ Yimsamran, Surapon 794 \\ Yip, Hwee S. 804 \\ Yokosawa, Jonny 1013 \\ Yoon, In-Kyu 1081 \\ Yoon, Sung Ho 119 \\ Yori, Pablo P. 869 \\ Young, Ginger R. 474 \\ Younis, Brima M. 1146 \\ Yu, Sun N. 717 \\ Yun, Na-Ra 119, 1206
}

Zahid, M. M. 345

Zaidi, Anita K. M. S1, S3, S49

Zalwango, Sarah 169

Zapor, Michael 501

Zarroug, Isam M. A. 51

Zhang, Cui 1006

Zhang, Liqin 123

Zhang, Wen-Yi 1006

Zhang, Xiuchun 123

Zhang, Zhiwen 1088

Zheng, Yan 138

Zhou, Guofa 1122

Zhou, Huayun 184

Zhou, Xiao-Nong 23

Zhu, Guoding 184

Ziperstein, Joshua 16

Zoerhoff, Kathryn L. 407 